\title{
Design, fabrication and experimental validation of a MEMS periodic auxetic structure
}

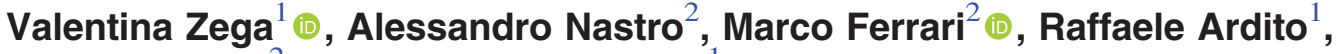 \\ Vittorio Ferrari $^{2}$ and Alberto Corigliano ${ }^{1}$ (i) \\ ${ }^{1}$ Department of Civil and Environmental Engineering, Politecnico di Milano, Milano, Italy \\ ${ }^{2}$ Department of Information Engineering, University of Brescia, Brescia, Italy \\ E-mail: valentina.zega@polimi.it
}

Received 18 January 2019, revised 14 May 2019

Accepted for publication 8 July 2019

Published 2 August 2019

\begin{abstract}
Auxetic structures are attracting increasing interest because of their large variety of applications at both the macro and micro scales. In this work, an innovative auxetic unit cell is presented, numerically studied, fabricated at the microscale and experimentally tested. Its versatility in terms of stiffness and Poisson's ratio and its compatibility with commercial Micro ElectroMechanical Systems (MEMS) fabrication processes suggest applications such as motion conversion and amplification mechanism in MEMS devices or on-chip biaxial tests. A MEMS auxetic and periodic structure exhibiting an overall equivalent Poisson's ratio lower than -1 is then proposed. The static and dynamic behaviours are studied both numerically and through a simplified analytical model. Experimental results confirm the simulated behaviour of the structure thus verifying the first MEMS auxetic structure with electrostatic actuation and capacitive readout.
\end{abstract}

Keywords: auxetic structure, MEMS, motion conversion, modelling

\section{Introduction}

Mechanical metamaterials have gained great attention in the last few decades due to the possibility to exploit unprecedented properties in various applications. Great attention is in particular addressed to the tuning, through mechanical design, of the Poisson's ratio of materials and structures. The Poisson's ratio is defined as the negative of the ratio of transverse strain to axial strain and can assume values from -1 to 0.5 for isotropic materials [1]. Most materials have Poisson's ratio between 0 and 0.5 where 0.5 means a perfect incompressible material and 0 refers to a material that does not exhibit any Poisson's effect.

On the other side, auxetic mechanical metamaterials $[2,3]$ are identified by a negative Poisson's ratio and are perhaps the most widely studied type of mechanical metamaterials because of the wide variety of possible applications either at the macro- and at the micro-scale. Earthquake protection [4], aerospace devices [5], and more specific applications which combine also the wave propagation control [6-13] are only some examples of applications at the macro-scale. At the micro-scale, auxetic structures are presented to experimentally test their negative Poisson's ratio property in micro-fabricated structures [14-16], and to discuss advanced properties by means of several numerical studies [17-19]. The actual use of these structures in microdevices, i.e. in the Micro Electro-Mechanical Systems (MEMS) world [20], is still an open issue due to the difficulty in the design process and in the interface with electronics, while it already happened for other types of metamaterials such as phononic crystals [21-27].

This work aims to pave the way to the application of the proposed auxetic structure in the MEMS field. The first possible application is the use of the auxetic structure as motion conversion mechanism [28, 29] in MEMS gyroscopes. MEMS gyroscopes are devices properly designed to measure the external angular rate by exploiting the Coriolis apparent force 


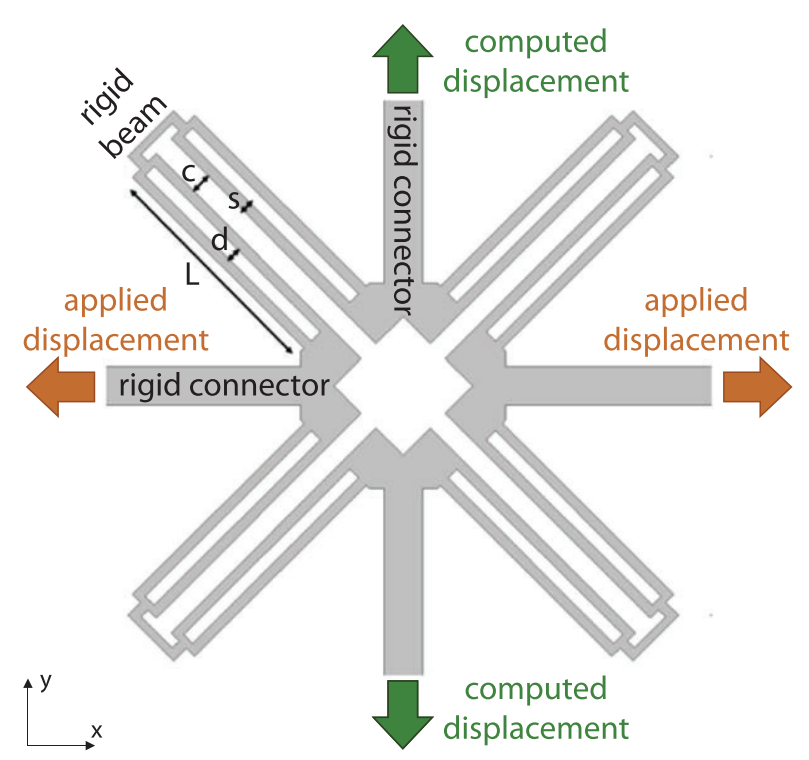

(a) Undeformed unit cell.

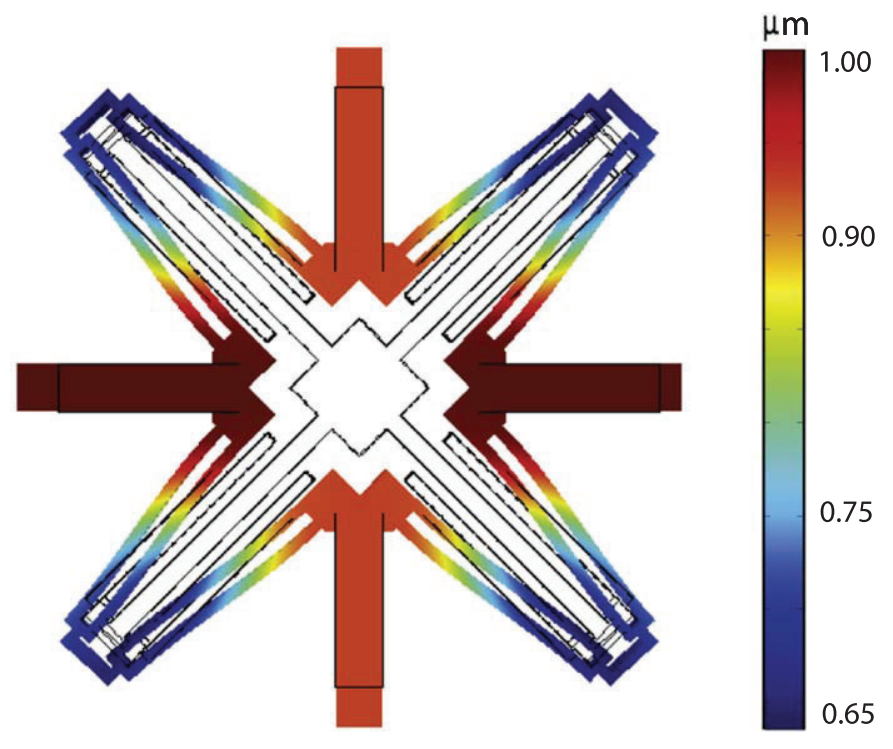

(b) Deformed unit cell.

Figure 1. Schematic view of the auxetic unit cell in the (a) unreformed and (b) deformed configurations.

that induces a displacement in the direction orthogonal to both the driving motion of the masses and the direction along which the external angular rate is applied [20, 30]. In multi-axial gyroscopes the simultaneous motion of the masses in two orthogonal directions is mandatory to exploit the Coriolis forces acting along different directions. At the same time, power consumption is a key aspect and consequently, MEMS designers need smart designs of coupling springs mechanisms that allow the desired motion of the masses without the need to actuate all of them separately [31]. Another possible application of the proposed auxetic structure in the MEMS world is represented by the on-chip biaxial tests [32, 33].

For the above purposes and to facilitate the integration with standard MEMS devices, the proposed design fulfils all the MEMS fabrication processes constraints (e.g. planarity) in particular the ones of the Thick Epitaxial Layer for Microgyroscopes and Accelerometers $\left(\right.$ ThELMA $^{\odot}$ ) surface micromachining process of STMicroelectronics [34] and can be actuated and detected through electrostatic schemes only.

The periodic structure shown in this work is designed starting from an innovative auxetic unit cell that allows the obtainment of an equivalent macroscopic Poisson's ratio lower than -1 . This is an interesting property for the application of the structure in MEMS devices as motion conversion and amplification mechanism. Mechanical amplification of motion is in fact a desired property in MEMS devices since it provides higher performances (i.e. lower noise for large displacements) without a higher power consumption. Moreover, the versatility of the proposed auxetic unit cell enables the possibility to exploit different negative Poisson's ratios starting from the same geometry: a MEMS designer can then tune the geometric dimensions according to his needs.

The paper is organized as follows. In section 2 , the innovative auxetic unit cell is presented, via the characteristics of its fundamental building blocks: parametric studies to exploit the negative Poisson's ratio property are presented and discussed. In section 3 an auxetic structure designed starting from the unit cell shown in section 2 is presented and its static and dynamic behaviour is studied in section 4 through both numerical simulations and a simplified one-degree-of-freedom model. Experimental tests are conducted in section 5 to show the negative Poisson's behaviour of the first MEMS compatible auxetic device controlled only via electrostatic schemes. Closing remarks are contained in section 6.

\section{Mechanical design of an auxetic unit cell}

The proposed auxetic unit cell consists of four folded beams inclined at $45^{\circ}$ with respect to the reference system and connected one to the other through rigid connectors as shown in figure 1(a). Each fold of the four inclined beams is composed of two parallel arms connected through a rigid perpendicular beam on one side and through the rigid connectors on the other side. When a displacement is applied in the $x$-direction to the two rigid connectors as shown in figure 1(a), the unit cell expands in the $y$-direction thus exhibiting an auxetic behaviour (figure 1(b)). A parametric analysis is here reported to show how it is possible to calibrate the negative macroscopic Poisson's ratio $\nu$ of the unit cell until reaching the ideal value of -1 only playing with the geometric dimensions of the unit cell.

\subsection{Parametric study of the auxetic cell}

The unit cell shown in figure 1 with geometrical parameters $L=60 \mu \mathrm{m}, c=5 \mu \mathrm{m}, d=3 \mu \mathrm{m}$ and $s=3 \mu \mathrm{m}$ is taken as reference in the following analyses. A constant out-of-plane thickness of $24 \mu \mathrm{m}$ is also considered. The stiffness $k_{\text {ref }}$, computed as the ratio between the reaction force on the $x$-axis 


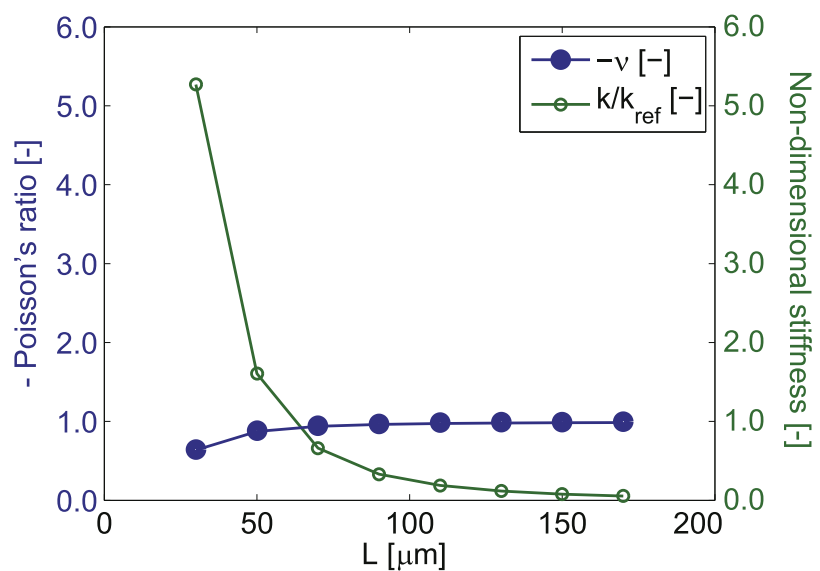

(a)

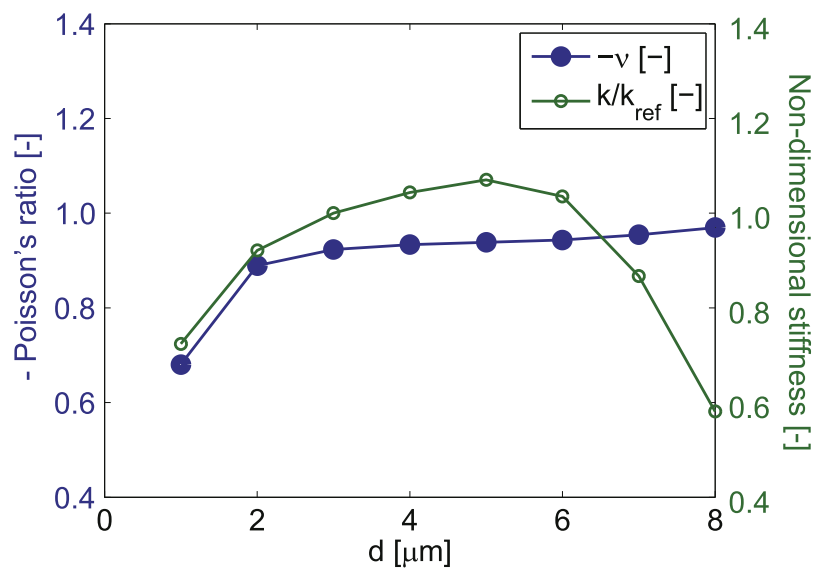

(c)

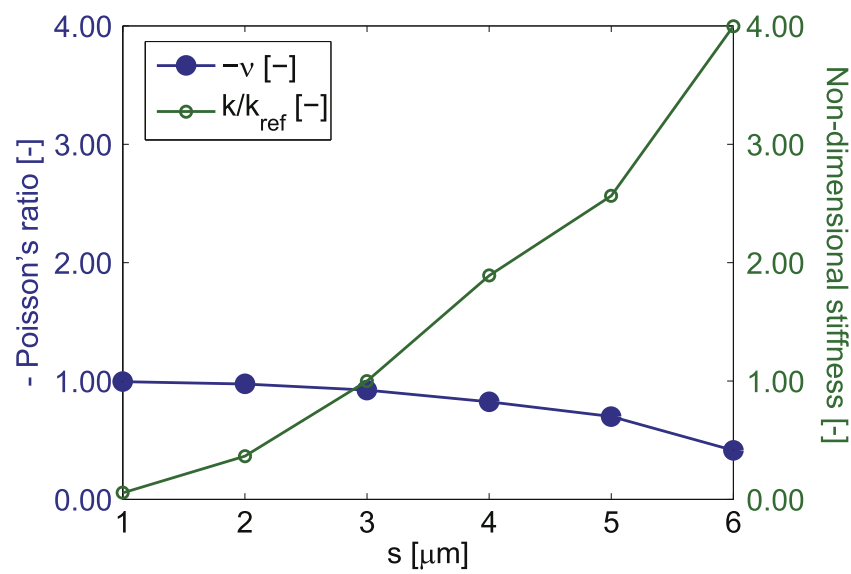

(b)

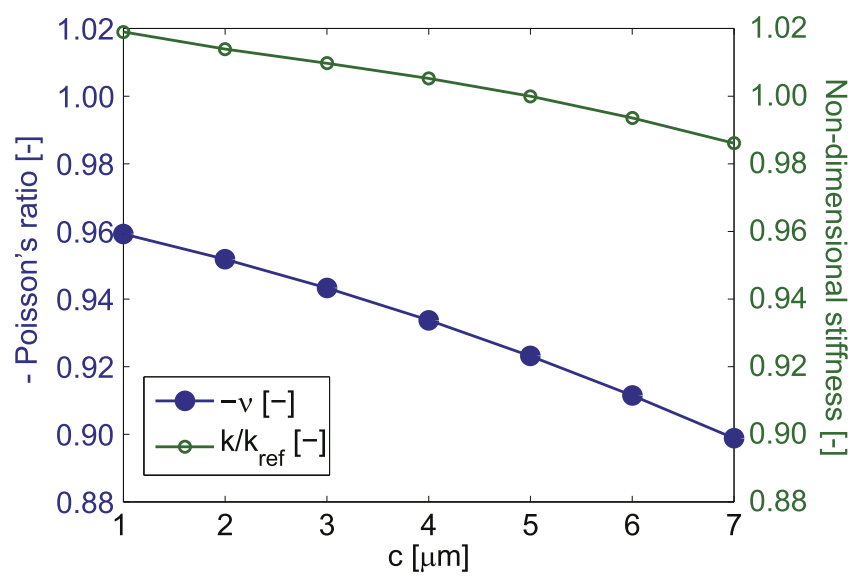

(d)

Figure 2. Macroscopic Poisson's ratio $(-\nu)$ and mechanical stiffness of the auxetic unit cell $\left(k / k_{r e f}\right)$ as a function of the geometrical parameters $L(\mathrm{a}), s(\mathrm{~b}), d$ (c) and $c$ (d) of the unit cell.

and the applied displacement in the same direction through a linear static numerical simulation in COMSOLMulthiphysics ${ }^{\odot}$, reads $1294 \mathrm{~N} \mathrm{~m}^{-1}$ and will be taken as a reference value. The four geometric parameters shown in figure 1 are changed independently one from the others and the computed macroscopic Poisson's ratios and mechanical stiffnesses are reported in figure 2.

From figure 2, it is clear that the macroscopic Poisson's ratio of the structure is negative for all the possible combinations of the geometric dimensions of the unit cell. Moreover, it is evident how exploring a wide range of stiffnesses, it is possible to obtain the macroscopic Poisson's value very close to the ideal limit -1 . The MEMS designer must then choose the geometric dimensions according to its specific requirements in terms of stiffness and Poisson's ratio and making an inevitable trade-off with the overall footprint of the structure.

\section{A micro-electro-mechanical auxetic structure}

Starting from the geometry of the unit cell studied in section 2, a MEMS auxetic periodic structure is here proposed. The overall auxetic behaviour is achieved through a
$5 \times 3$ repetition of the unit cell (figure 3(a)) while electrodes, located in properly designed frames, are employed for the displacement actuation and readout.

MEMS structures, because of their small dimensions (i.e. in the order of $\mu \mathrm{m}$ ) usually exploit electrostatic forces to actuate and detect the motion of their components [20].

In an electrostatic scheme based on parallel plates, one plate is fixed (i.e. anchored on the substrate) while the other is free to move parallel to the other (i.e. rigidly connected to the moving mass of the device). The capacitance measured between the two plates at rest position neglecting the effect of fringing fields, reads:

$$
C=\frac{\epsilon_{0} A}{g},
$$

where $\epsilon_{0}$ is the vacuum permittivity, $A$ is the facing area of the two plates and $g$ is the gap between them. Applying a displacement $y$ to the moving plate, connected to the mass of the MEMS structure, a reduction of the gap is produced, thus leading to a capacitive variation equal to:

$$
\frac{\mathrm{d} C}{\mathrm{~d} y}=\frac{\mathrm{d}}{\mathrm{d} y}\left[\frac{\epsilon_{0} A}{g-y}\right]=\frac{\epsilon_{0} A}{(g-y)^{2}} \simeq-\frac{\epsilon_{0} A}{g^{2}}
$$




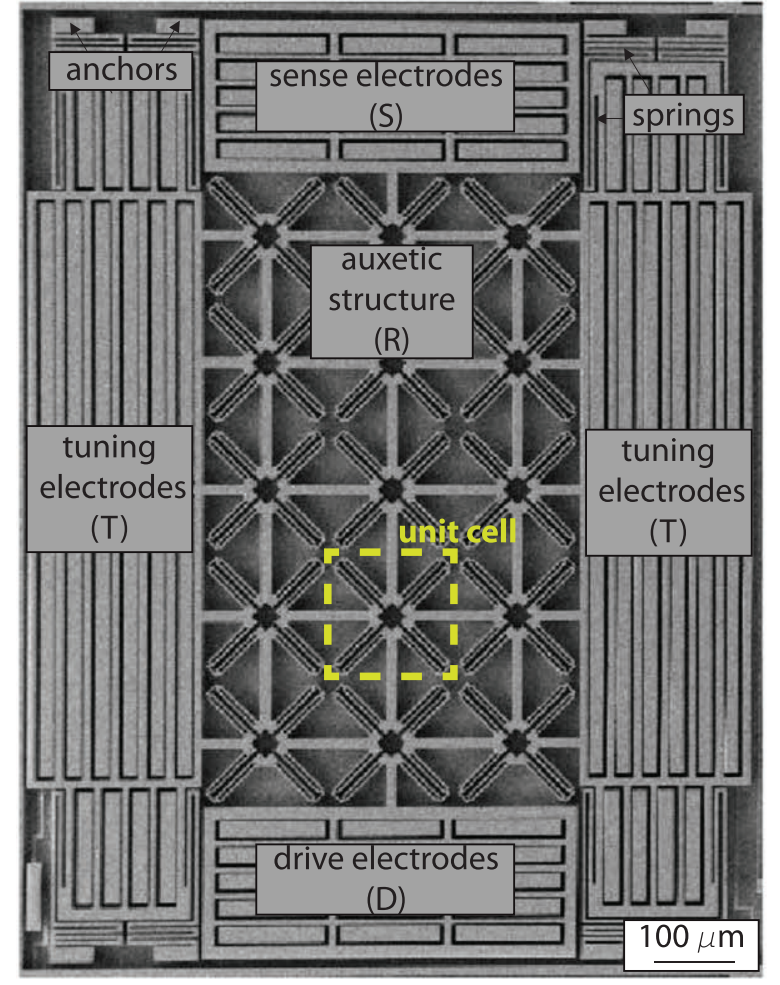

(a)
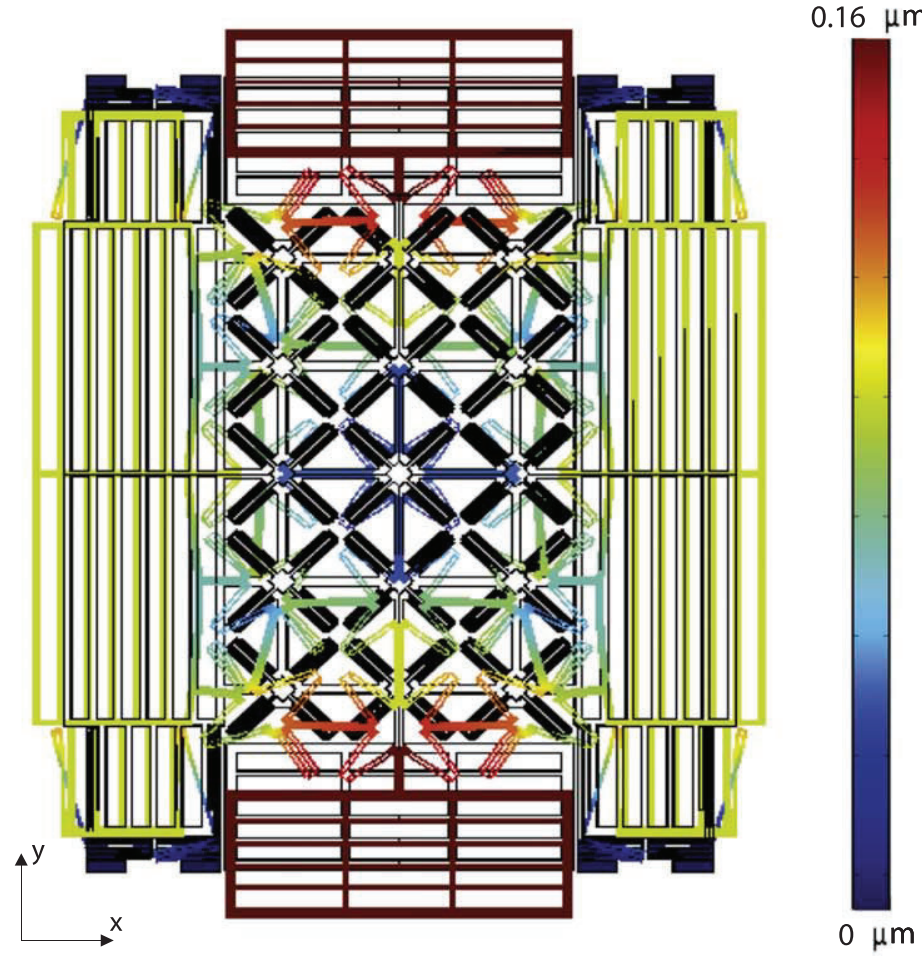

(b)

Figure 3. (a) Scanning electron microscope (SEM) image of the fabricated auxetic structure. (b) Auxetic behaviour of the structure: a displacement of $\pm 0.1 \mu \mathrm{m}$ has been applied on the right and left tuning electrodes, respectively.

and consequently the displacement of the structure can be detected by the electronic circuit connected to the capacitor. Note that in the last approximation of equation (2), small displacements $y \ll g$ are considered.

The electrostatic scheme based on parallel plates capacitors can be also employed to apply a force on the mass (i.e. to exploit the auxetic behaviour). It reads:

$$
F_{e l} \simeq-\frac{1}{2} \frac{\epsilon_{0} A}{g^{2}} \Delta V^{2},
$$

where $\Delta V$ is the voltage difference between the fixed and the moving electrodes.

A set of electrodes (called tuning electrodes in figure 3(a)) are then located at the left and right sides of the auxetic structure to apply the tensile force needed to exploit the auxetic behaviour, while other electrodes (called sense and drive electrodes in figure 3(a)) are on the sense and drive frames to detect the displacements induced in the $y$-direction by the applied force.

Finally folded springs are employed to suspend the MEMS structure as shown in figure 3(a). Despite these folded springs are less stiff than the central auxetic structure, they must be considered in the simulations and in the theoretical prediction of the static and dynamic behaviour of the structure.

In figure 3(b) the auxetic deformation of the proposed structure is shown. Because of the complexity of the structure and thanks to border effects (e.g. rotations) that happen in the corner unit cells, it is possible to obtain a Poisson's ratio much lower than -1 as it will be described in the next section.

\section{Fabricated structure and numerical simulations}

A prototype of the MEMS auxetic structure has been fabricated through the ThELMA ${ }^{\odot}$ surface micromachining process developed by STMicroelectronics. It is made in polysilicon (Young's modulus and density equal to $169 \mathrm{GPa}$ and $2330 \mathrm{~kg} \mathrm{~m}^{-3}$, respectively) and has an out-of-plane constant thickness equal to $24 \mu \mathrm{m}$ according to the fabrication process constraints. The geometrical parameters of the auxetic unit cell of the fabricated device are collected in table 1 .

The over etch, which is defined as a deviation between the designed masks and the effective dimensions of the suspended parts is here considered constant and equal to the nominal value of the employed fabrication process (i.e. $0.4 \mu \mathrm{m}$ ) for the in-plane dimensions while no over etch is considered for the out-of-plane thickness. This is an over simplified hypothesis that can lead to small discrepancies between theoretical values and experimental data since nonhomogeneities can arise during the MEMS fabrication process. Being the purpose of the paper to prove the auxetic behaviour of the proposed MEMS structure, we adopt this assumption for the sake of simplicity.

Starting from the real geometry of the MEMS auxetic structure and considering the simplifying hypotheses on the 
Table 1. Geometric dimensions of the auxetic unit cell of the fabricated structure shown in figure 3(a). A nominal over etch of $0.4 \mu \mathrm{m}$ is considered for the in-plane dimensions.

\begin{tabular}{lc}
\hline$L$ & $62.37 \mu \mathrm{m}$ \\
$s$ & $2.88 \mu \mathrm{m}$ \\
$d$ & $3.00 \mu \mathrm{m}$ \\
$c$ & $5.18 \mu \mathrm{m}$ \\
\hline
\end{tabular}

over etch, numeric simulations are performed in COMSOLMulthiphysics ${ }^{\circledR}$ to estimate both the static and dynamic behaviour of the structure and the expected equivalent Poisson's ratios.

\subsection{Static behaviour}

An asymmetric auxetic behaviour along the two orthogonal in-plane directions is expected for the whole structure, due to its geometry (i.e. $5 \times 3$ unit cells scheme) and to the presence of other mechanical elements (i.e. frames, suspension springs).

First, a force is applied on the tuning electrodes of the structure simulating the electrostatic actuation and the obtained displacements are computed on all the frames through a static analysis. Linear elastic simulations are performed since the entity of the forces statically applied through the tuning electrodes are small enough not to enter into the nonlinear regime (i.e. computed displacements in the order of some tens of $\mathrm{nm}$ for the voltages of section 5.1). The stiffness is equal to $4444 \mathrm{~N} \mathrm{~m}^{-1}$, while the equivalent Poisson's ratio defined as the ratio between the displacements of the sense/ drive frame and the displacements of the tuning electrodes, is equal to -1.6 .

If now the force is applied on both the sense and drive electrodes, the second equivalent Poisson's ratio and mechanical stiffness of the structure can be estimated: they are equal to -0.27 and $781.88 \mathrm{~N} \mathrm{~m}^{-1}$, respectively.

\subsection{Dynamic behaviour}

Two modes are taken into account for the dynamic characterization of the MEMS auxetic structure: an in-plane translation of the structure along the $y$-direction (figure 4(a)) and the 'auxetic' mode (figure 4(b)).

A modal analysis of the structure is performed in COMSOLMulthiphysics $^{\circledR}$ to compute the natural frequencies of the two modes of interest that read $31633 \mathrm{~Hz}$ and $62140 \mathrm{~Hz}$, respectively.

To obtain a theoretical estimation of the frequency response of the MEMS auxetic structure, a one degree of freedom model is considered. This represents a very simplified model to describe the overall dynamic behaviour of the structure. A more complex numerical model able to couple the mechanical and electrostatic physics is necessary if one wants to describe the real behaviour of the proposed MEMS auxetic structure in a quantitative way, in particular when the large displacement regime is entered.
Note that, according to the measurements that will be shown in section 5.2, in the following the drive and sense electrodes are kept at DC-value equal to zero and used to actuate and detect the modes, respectively, while the tuning electrodes are kept at the same potential $V$ of the auxetic structure to avoid any kind of electrostatic force in the $x$-direction. A sinusoidal signal $v_{p}(t)=v_{p_{a}} \cos \left(\omega_{0} t\right)$ of small amplitude (i.e. $v_{p_{a}} \ll V$ ) at the frequency $\omega_{0}$ of the mode of interest is applied between the drive electrodes and the auxetic structure $\left(\Delta V=V-v_{p}(t)\right)$ for the actuation.

The electrostatic force exerted by the drive electrodes on the structure reads [20]:

$$
F_{e l}^{d r}(t)=\frac{\epsilon_{0} A_{d r}}{g_{d r}^{2}} V v_{p}(t)+\frac{\epsilon_{0} A_{d r}}{g_{d r}^{3}} V^{2} y(t),
$$

where a Taylor expansion up to the first order has been performed while higher order components have been neglected. Note that, according to the actuation and detection schemes, the $y$-axis rigid displacement $y(t)$ of the sense and drive frames is considered as the single degree of freedom of the system. An expression similar to equation (4) can be found for the electrostatic force exerted by the sense electrode on the MEMS structure by putting $v_{p}(t)=0$ and substituting the sensing area and gap to the driving counterparts. Note that the gap between the driving (sensing) electrodes and the moving mass can decrease or increase according to the mode under study. In the following the $y$-axis translational mode is considered for the sake of simplicity.

The equation of motion then reads:

$$
m \ddot{y}(t)+b \dot{y}(t)+k y(t)=F_{e l}^{t o t}(t),
$$

where $m$ is the modal mass of the structure computed from the modal analysis, $b$ is the damping factor experimentally measured (see section 5.2), $k$ is the mechanical stiffness computed through a static analysis and $F_{e l}^{t o t}(t)$ is the total electrostatic force exerted on the structure. By substituting equation (4) and the force exerted on the auxetic structure by the sensing electrodes into equation (5), one obtains:

$$
m \ddot{y}(t)+b \dot{y}(t)+k_{1} y(t)=F(t),
$$

where:

$$
\begin{aligned}
k_{1} & =k_{1_{m}}-k_{1_{e l}}=k_{1_{m}}-\left(\epsilon_{0} \frac{A_{d r}}{g_{d r}^{3}} V^{2}+\frac{\epsilon_{0} A_{\text {sen }}}{g_{\text {sen }}^{3}} V^{2}\right) \\
F(t) & =\frac{\epsilon_{0} A_{d r}}{g_{d r}^{2}} V v_{p}(t) .
\end{aligned}
$$

The linear mechanical stiffness $k_{1_{m}}$ of the translational mode along the $y$-direction is found to be equal to $266.86 \mathrm{~N} \mathrm{~m}^{-1}$, while the stiffness of the 'auxetic' mode is equal to $781.88 \mathrm{~N} \mathrm{~m}^{-1}$ as shown in section 4.1. As expected, the parallel plates scheme provide a negative linear electrostatic stiffness that induces a softening effect on the structure, thus lowering the natural frequency computed through the modal analysis (see figure 4 and table 2, II column).

By solving equation (6) and considering the harmonic driving term $F(t)=F_{a} \cos (\omega \mathrm{t})$, of frequency $\omega$ close to the natural frequency of the translational and auxetic modes, it is 


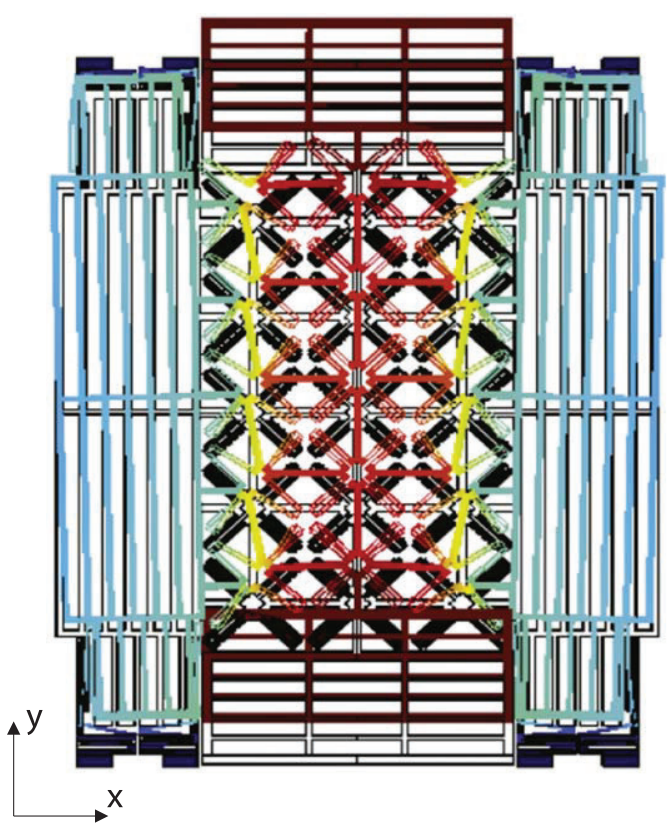

(a) $f=31633 \mathrm{~Hz}$

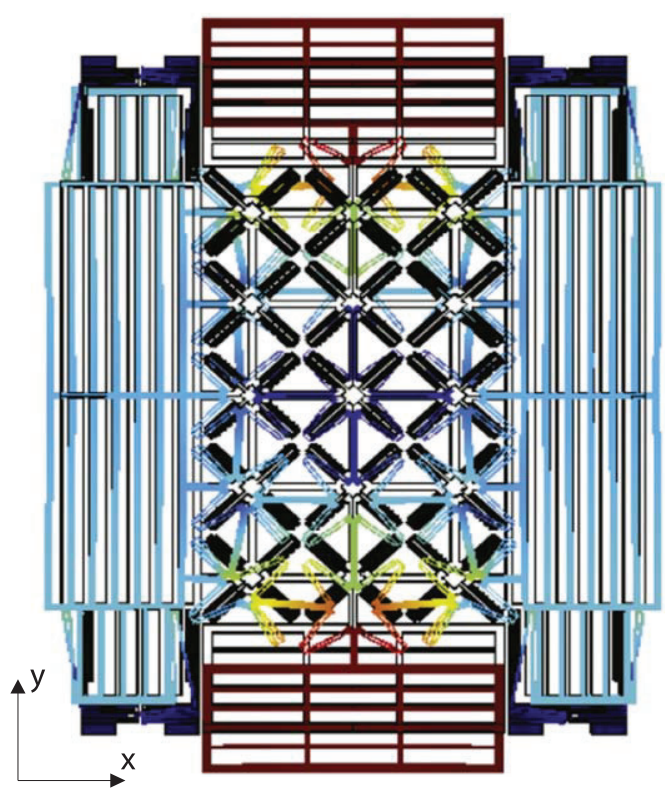

(b) $f=62140 \mathrm{~Hz}$

Figure 4. Modal analysis performed in COMSOLMultiphysics ${ }^{\circledR}$. (a) $Y$-axis translational and (b) auxetic modes. The contour of the displacement field is shown in colour.

Table 2. Comparison between experimental and numerical natural frequencies of two modes of the auxetic structure. Numerical frequencies are computed through a FEM modal analysis performed in COMSOLMultiphysics ${ }^{\circledR}$ and take into account the electrostatic softening induced by the bias voltage applied on the structure.

\begin{tabular}{lccc}
\hline & FEM & Experiments & $\Delta=($ Exp - FEM $) /$ Exp $\cdot 100$ \\
\hline Translational mode & $31399 \mathrm{~Hz}$ & $31050 \mathrm{~Hz}$ & $-1.12 \%$ \\
Auxetic mode & $62006 \mathrm{~Hz}$ & $61090 \mathrm{~Hz}$ & $-1.41 \%$ \\
\hline
\end{tabular}

possible to obtain a relation between the amplitude $F_{a}$ and the frequency $\omega$ of the driving force and the amplitude $Y$ of the forced vibration of the system:

$$
\left(\frac{F_{a}}{k_{1}}\right)^{2}=\left(2\left(1-\frac{\omega}{\omega_{0}}\right) Y\right)^{2}+\left(\frac{b}{m \omega_{0}} Y\right)^{2},
$$

where $\omega_{0}$ is the natural frequency of the mode under study.

When the large displacement regime is entered, the analytical model (5) must be enriched with nonlinear terms coming from the mechanical response of the structure and/or the electrostatic forcing. The nonlinear equation of motion, describing the dynamics of the equivalent one-degree-offreedom model, assumes then the expression:

$$
m \ddot{y}(t)+b \dot{y}(t)+k(y(t)) y(t)=F_{e l}^{t o t}(t),
$$

where $k(y(t))$ is the nonlinear mechanical stiffness and the total electrostatic force is computed as the sum of the two contributions coming from the driving and sensing electrodes. The electrostatic force exerted by the driving electrodes on the structure expanded in Taylor's series up to the third order reads [20]:

$$
\begin{aligned}
F_{e l}^{d r}(t)= & \frac{\epsilon_{0} A_{d r}}{g_{d r}^{2}} V v_{p}(t)+\frac{A_{d r} \epsilon_{0}}{g_{d r}^{3}} V^{2} y(t) \\
& +\frac{3}{2} \frac{A_{d r} \epsilon_{0}}{g_{d r}^{4}} V^{2} y(t)^{2}+2 \frac{A_{d r} \epsilon_{0}}{g_{d r}^{5}} V^{2} y(t)^{3},
\end{aligned}
$$

while the expression of the electrostatic force exerted by the sensing electrodes can be computed by putting $v_{p}(t)=0$ and substituting the sensing area and gap to the driving counterparts. Note that the III order term in the Taylor expansion (10) is enough to catch the nonlinear behaviour of the full expression of the electrostatic force in the range of displacement experienced in the experiments that will be discussed in section 5.2. In particular the nonlinear mechanical stiffness can be expanded in Taylor's series up to the third order (i.e. $\left.k(y(t))=k_{1_{m}}+k_{2_{m}} y(t)+k_{3_{m}} y(t)^{2}\right)$. By combining the nonlinear terms coming from the mechanical stiffness with the Taylor expansion of the total electrostatic force (i.e. $\left.F_{e l}(t)=F(t)+k_{1_{e l}} y(t)+k_{2_{e l}} y(t)^{2}+k_{3_{e l}} y(t)^{3}\right)$, it is possible to re-write equation (9) in the form:

$$
m \ddot{y}(t)+b \dot{y}(t)+k_{1} y(t)+k_{2} y(t)^{2}+k_{3} y(t)^{3}=F(t),
$$




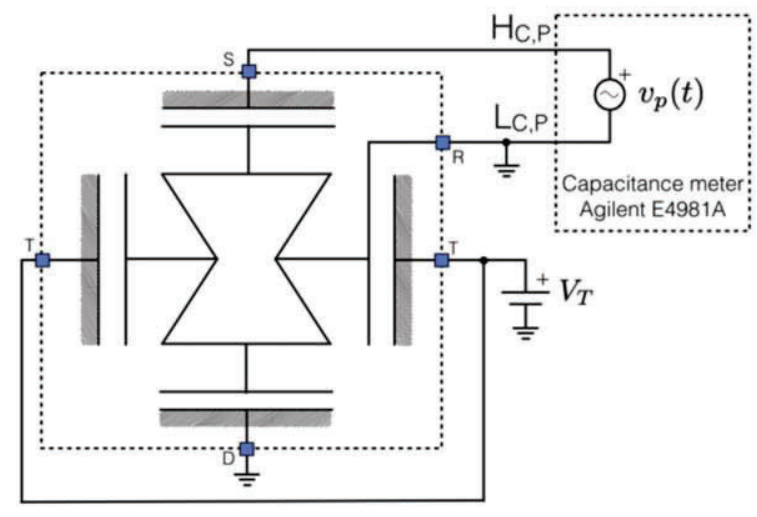

(a)

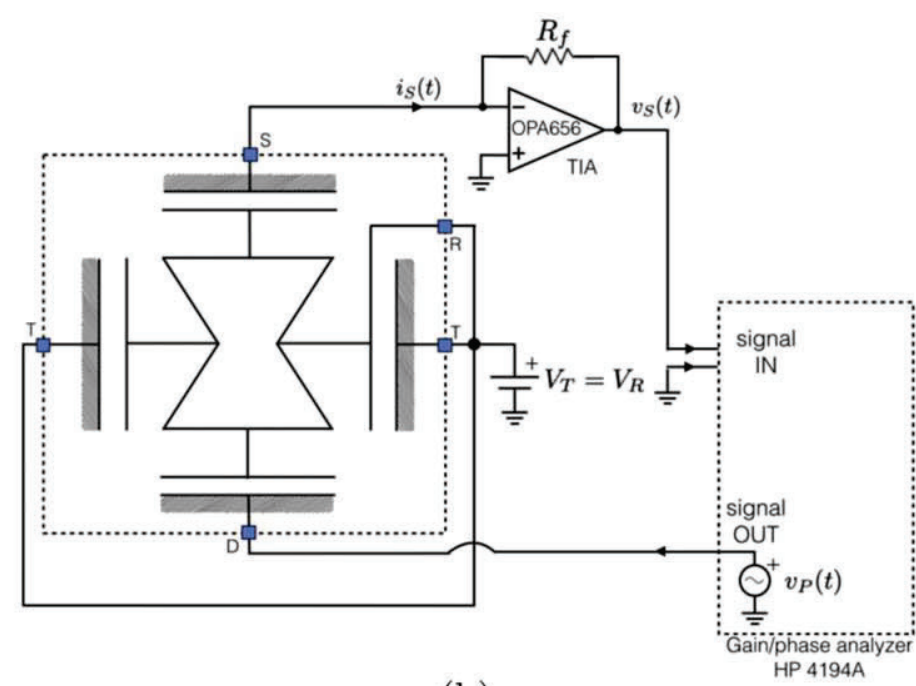

(b)

Figure 5. Block diagram of the setup employed for the static (a) and the dynamic (b) characterization.

where

$$
\begin{aligned}
& k_{1}=k_{1_{m}}-k_{1_{e l} l}, \\
& k_{2}=k_{2_{m}}-k_{2_{e l},} \\
& k_{3}=k_{3_{m}}-k_{3_{e l}} .
\end{aligned}
$$

The presence of quadratic and cubic terms of different nature in equation (11) suggests that a hardening or softening response can be obtained depending on the polarization conditions and on the entity of the displacements. Since the simulation of the mechanical nonlinear dynamic behaviour of the structure requires the development of a proper simulation tool that lies outside the purpose of this paper, in the following only electrostatic nonlinearities will be considered (i.e. $k_{2_{m}}=k_{3_{m}}=0$ ).

Applying the method of successive approximations (see $[35,36]$ for more details) and considering the harmonic driving term $F(t)=F_{a} \cos (\omega \mathrm{t})$, of frequency $\omega$ close to the natural frequency of the mode of interest, it is possible to obtain a relation between the amplitude $F_{a}$ and the frequency $\omega$ of the driving force and the amplitude $Y$ of the forced vibration of the system:

$$
\begin{aligned}
\left(\frac{F_{a}}{k_{1}}\right)^{2}= & \left(2\left(1-\frac{\omega}{\omega_{0}}\right) Y+\left(\frac{3}{4} \frac{k_{3}}{k_{1}}-\frac{5}{6}\left(\frac{k_{2}}{k_{1}}\right)^{2}\right) Y^{3}\right)^{2} \\
& +\left(\frac{b}{m \omega_{0}} Y\right)^{2} .
\end{aligned}
$$

Note that, the method of successive approximations is only one of the possible approaches proposed in the literature for the solution of nonlinear dynamic systems. In [37], the harmonic balance method is for example proposed as a valid alternative, while in [38], the energy balance method is employed for the solution of a nonlinear dynamic system. Younis et al [39, 40] proved that perturbation methods [41] such as the method of multiple scales [42] provide better and more consistent solutions with respect to the others, especially in the highly nonlinear regime.
Another possibility for the solution of nonlinear dynamic equations, is the use of numerical techniques such as the continuation method with arc length control [43-45].

For the sake of completeness, we solved equation (11) through three different methods: perturbations, multiple scales and continuation and we found a very good agreement between the results in the range of displacement experienced in the experimental tests proposed in section 5.2. More discrepancies can arise if the highly nonlinear regime is entered.

\section{Experimental tests}

To experimentally validate the auxetic behaviour of the proposed MEMS device, tests in both static and dynamic regimes are performed [46]. Figures 5(a) and (b) show the block diagram of the experimental setup implemented to perform static and dynamic characterizations, respectively.

Figure 6(a) shows the capacitance meter Agilent E4981A and the gain-phase analyzer HP 4194A used to perform static and dynamic characterizations. Figures 6(b) and (c) show the auxetic MEMS device bonded inside a 68-pins plastic-leadedchip-carrier (PLCC) package and the printed circuit board used to test the device.

\subsection{Static measurements}

For the static characterization, both the capacitance $C_{D R}$ and $C_{S R}$ are measured, exploiting a capacitance meter. $C_{D R}$ refers to capacitance between $D$ and $R$, while $C_{S R}$ refers to the capacitance between $S$ and $R$. Furthermore, a DC-voltage $V_{T}$ is applied between $T$ and $R$ to induce a tensile force needed to exploit the auxetic behaviour. Figure 5(a) shows the block diagram of the experimental setup used to measure the capacitance $C_{S R}$, where $D$ is grounded and the sinusoidal probing signal $v_{P}(t)$ is applied between $S$ and $R$. A similar configuration is implemented to measure the capacitance $C_{D R}$, 


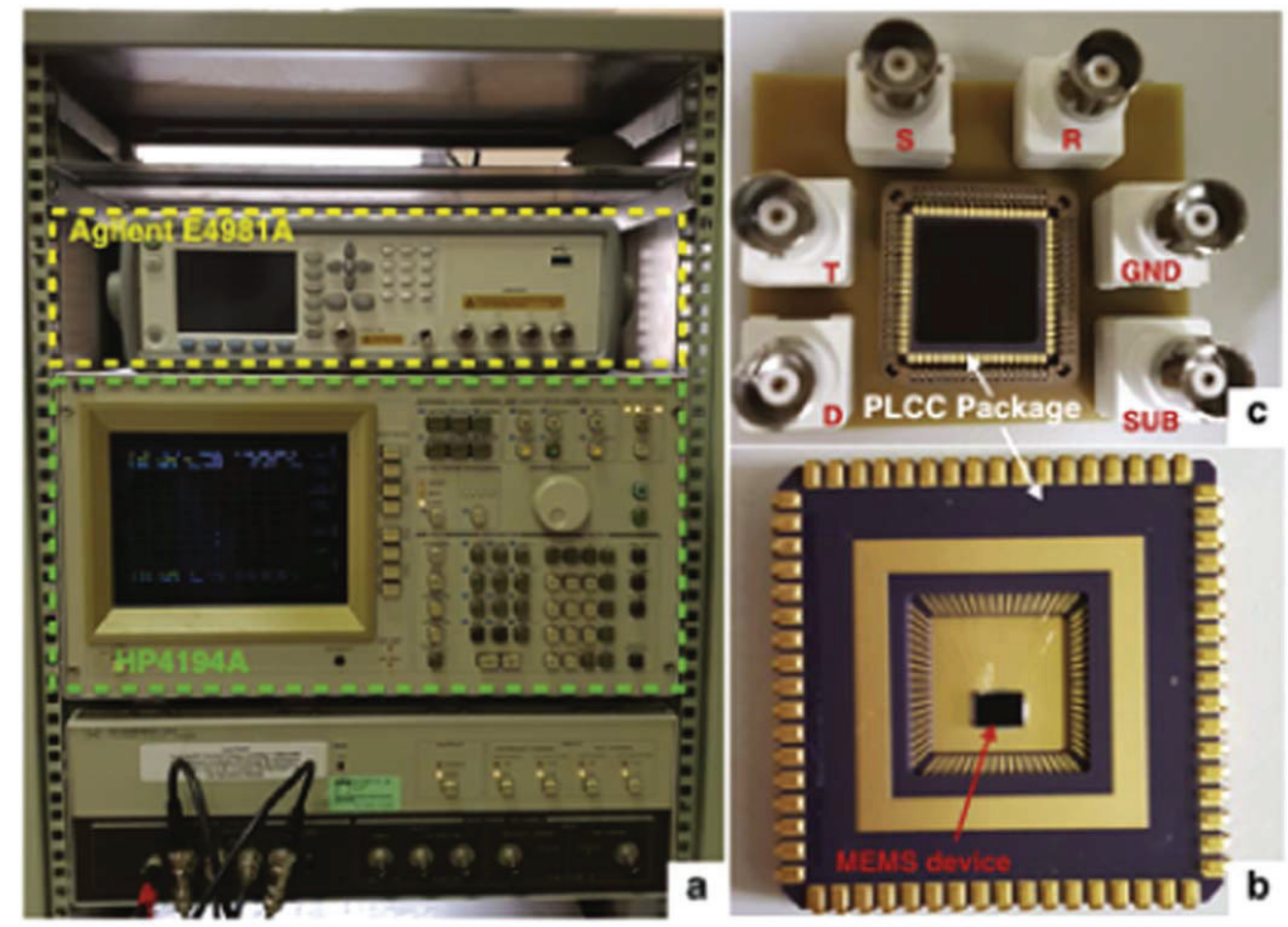

Figure 6. (a) Experimental setup with the gain-phase analyzer and the capacitance meter, (b) enlarged views of the auxetic MEMS bonded into the PLCC package and (c) of the printed circuit board used for static and dynamic measurements.

with the probing signal $v_{P}(t)$ applied between $D$ and $R$, with $S$ grounded. The peak amplitude and the frequency of the probing signal $v_{P}(t)$ are set to $100 \mathrm{mV}$ and $1 \mathrm{MHz}$, respectively, in order to produce negligible electrostatic force while maintaining good accuracy of the capacitance measurements.

In figure 7 , the experimental results are shown in comparison with the numerical predictions as discussed in section 4 under the simplifying hypotheses on the over etch. As expected from the auxetic behaviour, when a tensile force is applied on the tuning electrodes along the $x$-direction, the structure expands along the $y$-direction, thus causing an increase in capacitance in both sense and drive electrodes.

To further investigate the auxetic behaviour of the MEMS structure, we applied a constant voltage $V_{S}=V_{D}$ on the sense and drive electrodes simultaneously while keeping the structure at a constant voltage equal to zero (i.e. $\Delta V=V_{S}=V_{D}$ on sense and drive electrodes) and we measured the capacitance variation on the tuning electrodes. In figure 8 the results of measurements are reported together with numerical predictions. Also in this case, the agreement between experimental data and the theoretical predictions is good and the auxetic behaviour of the structure is well captured by the readout circuit. Note that, in agreement with the results of section 4 , the auxetic behaviour of the structure is not symmetric: the auxeticity is more pronounced if the voltage is applied on the tuning electrodes.

\subsection{Dynamic measurements}

For the dynamic characterization, the frequency response of the structure in proximity of the natural frequencies of two in-plane global modes is measured. The two modes of interest are an in-plane translation of the structure along the $y$-direction and the 'auxetic' mode (see figure 4 and the insets of figures 9(a) and (b), respectively).

A gain-phase analyzer is exploited to measure the frequency response between $D$ and $S$. The capacitance $C_{D R}$ is used as an electrostatic actuator by applying the sinusoidal probing signal $v_{p}(t)=v_{p_{a}} \cos \left(\omega_{0} t\right)$ to $D$, as reported in figure 5(b). A DC-voltage $V_{R}$ is applied to $R$ in order to bias the capacitor $C_{D R}$ and, at the same time, to provide the DCvoltage $V_{S R}$ to allow the measurement of the capacitance $C_{S R}$, used as a variable-gap capacitive sensor. The generated sinusoidal electrostatic force actuates the mechanical movable structure from $D$, producing a sinusoidal displacement at $S$ which is converted into variations of the capacitance $C_{S R}$ [46]. Thanks to the virtual ground of the transimpedance amplifier TIA (OPA656), the voltage at $S$ is kept equal to zero. Therefore, the sensing current $i_{S}(t)$ in $C_{S R}$ results:

$$
i_{S}(t)=-V_{S R} \frac{\mathrm{d} C_{S R}(t)}{\mathrm{d} t} .
$$

The current $i_{S}(t)$ is then converted into the voltage $v_{S}(t)$ by the TIA. In the frequency domain, the transfer function of the TIA is given by:

$$
\frac{V_{S}(f)}{I_{S}(f)}=-R_{f},
$$

where $R_{f}=100 \mathrm{k} \Omega$ is the feedback resistance. During the dynamic measurements, the DC-voltage $V_{T R}$ is kept equal to zero $\left(V_{T}=V_{R}\right)$ in order not to create a tensile force, thus not exploiting the auxetic behaviour of the structure. Moreover, 

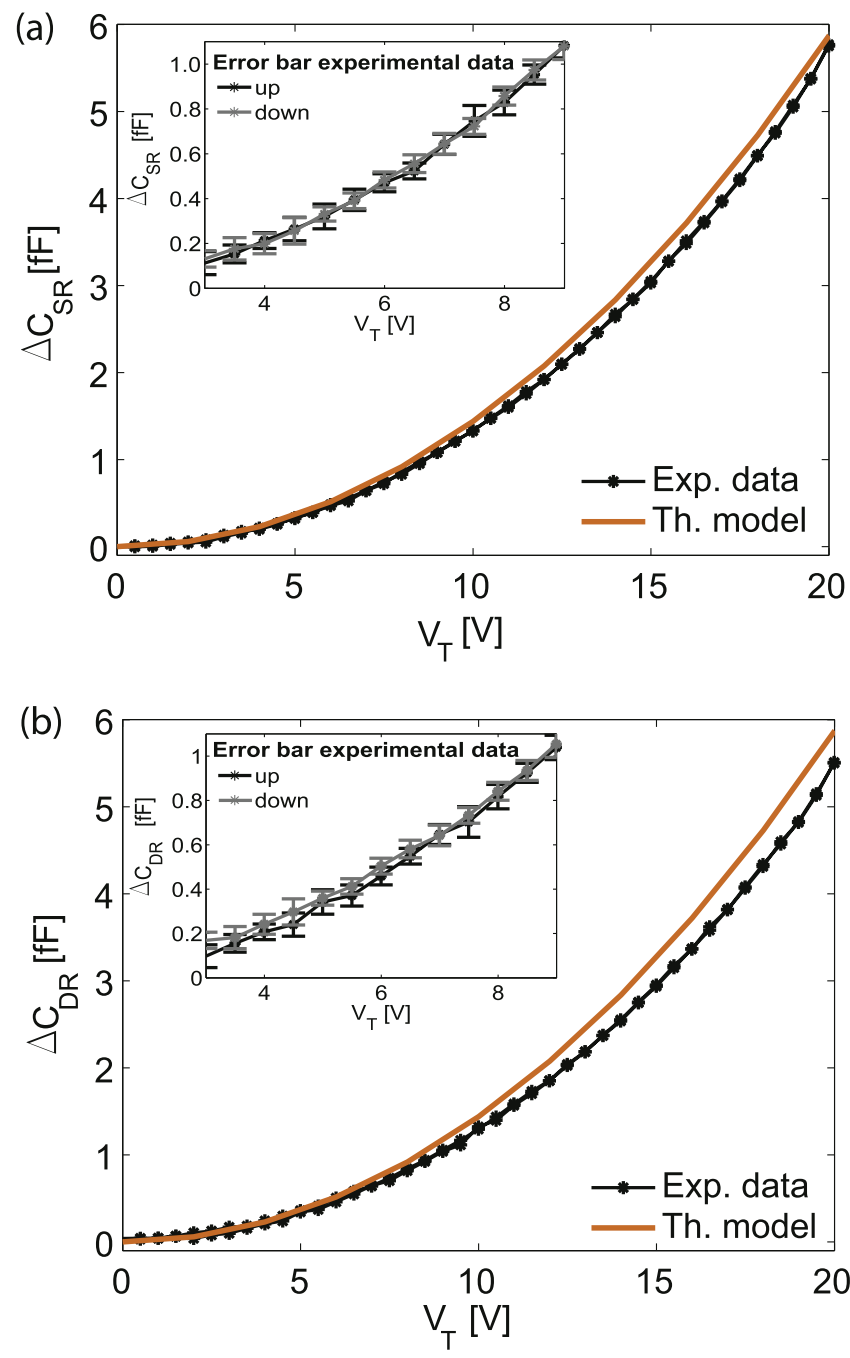

Figure 7. Capacitance variation versus applied voltage curves. An increasing voltage $V_{T}$ is applied on the tuning electrodes and the capacitance variation on the (a) sense $\left(\Delta C_{S R}\right)$ and (b) drive $\left(\Delta C_{D R}\right)$ electrodes is measured. The theoretical model is compared with the experimental data.

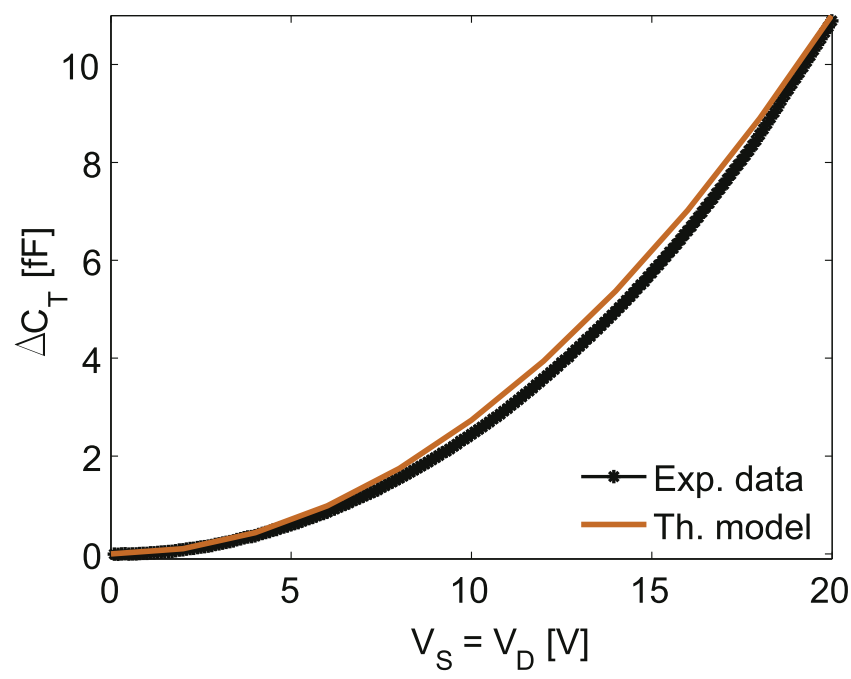

Figure 8. Capacitance variation measured on the tuning electrodes $\left(\Delta C_{T}\right)$ versus voltage applied on sense and drive electrodes. the parasitic capacitance is numerically compensated in order to eliminate the parallel resonant frequency of the transfer function $V_{S}(f) / V_{P}(f)$ of the structure [47, 48].

In figure 9 the experimental frequency responses of the structure for $V_{T}=V_{R}=5 \mathrm{~V}$ and a $v_{p_{a}}=9.95 \mathrm{mV}_{r m s}$ are reported together with the numerical predictions computed through equation (8). A quality factor $(Q)$ of about 1400 and 1700 for the $y$-axis translational mode and the auxetic mode are found, respectively.

In table 2 both the experimental and the numerical (i.e. obtained through a modal analysis and by taking into account the electrostatic softening effect induced by the polarized parallel plates as explained in section 4.2) natural frequencies of the two modes under study are reported.

The discrepancy between the numerical and the experimental frequencies is mainly due to the fabrication imperfections that are not considered in the model (i.e. constant inplane over etch). Moreover, the slightly different values of the relative percentage $\Delta$ reported in the IV column of table 2 , can be justified by the fact that the two modes involve the deformation of different springs of the structure: the auxetic mode exploits the deformation of the central unit cells while the translational one does not involve such beams.

Finally in figure 10 , the $y$-axis translational mode is tested in the nonlinear regime. The comparison between the analytical model in equation (15) and the experimental results is reported for three different values of the sinusoidal probing signal (i.e. $v_{p_{a}}=2.12,9.95,19.8 \mathrm{mV}_{r m s}$ ) for a $V_{R}=15 \mathrm{~V}$.

The overall softening behaviour is qualitatively catched by the simplified analytical model. To quantitatively describe the nonlinear experimental response of the structure, a numerical model able to couple the mechanics and the electrostatics is needed. The deviation between the experimental data and the theoretical predictions in the nonlinear regime can be indeed explained by the fact that in the analytical model: (i) the over etch is constant and equal to the nominal value of $0.4 \mu \mathrm{m}$, (ii) material parameters are known within a certain range, (iii) the electrostatic forces are considered as in equation (10) thus neglecting fringe fields and other nonidealities in the parallel plate scheme as for example the small rotation of the structure with respect to the fixed electrodes due to fabrication imperfections and (iv) an over-simplification is introduced by considering the MEMS auxetic structure as a one degree of freedom system. Finally, we want to stress the fact that mechanical nonlinearities are not taken into account in the model: as known from the literature they usually lead to hardening behaviour of the structure and consequently cannot improve the agreement between theoretical model and experimental results. However, in the auxetic unit cell (figure 1(a)) the deformable components are very similar to the coupling springs studied in [31] that exhibit a softening geometric nonlinearity if deflected. Further investigations in this direction are needed since the deformation of the auxetic structure according to the $y$-axis translational mode is very complicated (figure 4). 


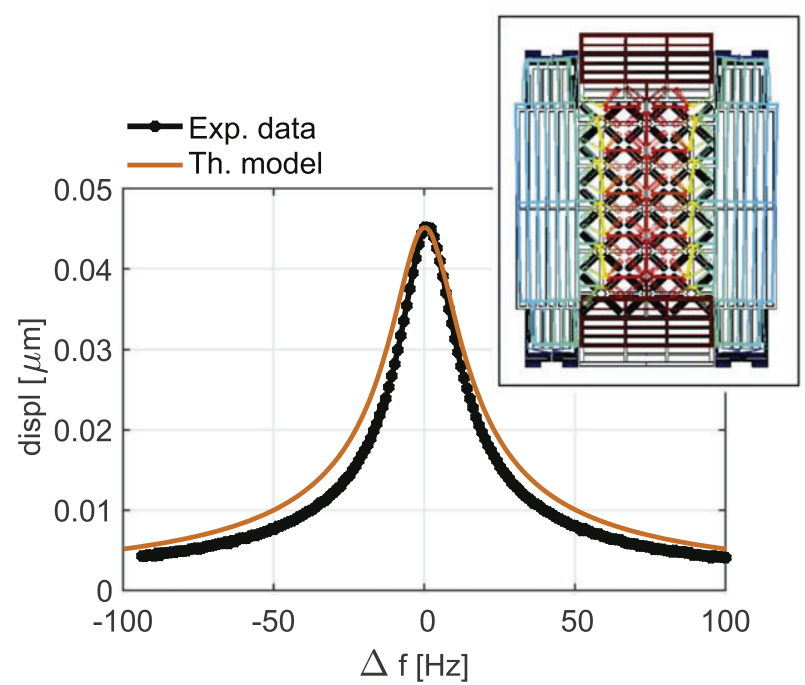

(a) Translational mode of the structure

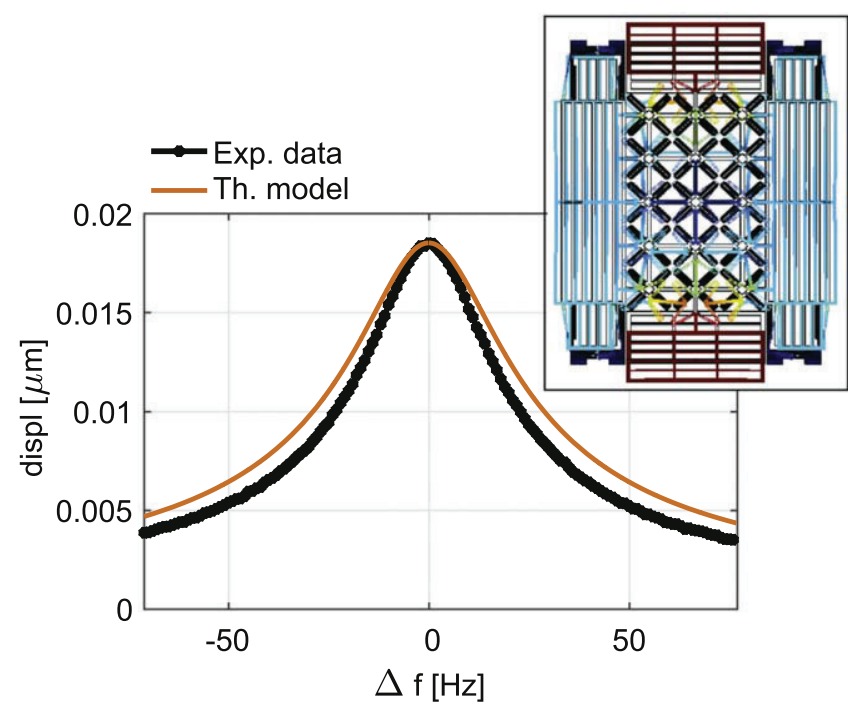

(b) Auxetic mode of the structure.

Figure 9. Experimental frequency response of the auxetic structure.

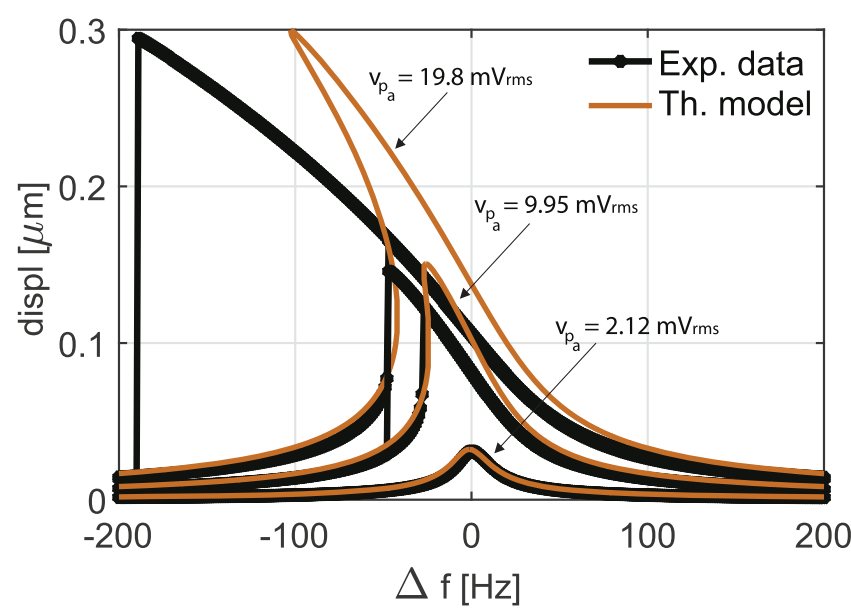

Figure 10. Frequency response of the MEMS auxetic structure. Comparison between experimental data and theoretical predictions for four different amplitudes (i.e. $v_{p_{a}}=2.12,9.95,19.8 \mathrm{mV}_{r m s}$ ) of the probing signal around the natural frequency of the $y$-axis translational mode and for $V_{R}=15 \mathrm{~V}$.

\section{Conclusions}

A new auxetic unit cell is proposed and its versatility is proved through a parametric analysis. A MEMS auxetic structure exhibiting a macroscopic equivalent Poisson's ratio lower than -1 is designed starting from this unit cell having in mind applications such as motion conversion and amplification mechanisms in MEMS devices or on-chip biaxial tests. Electrostatic actuation and capacitive detection schemes are employed to improve the integrability of the proposed structure with commercial MEMS devices (e.g. gyroscopes).

Numerical simulations are conducted to simulate the auxetic behaviour of the structure in statics and a simplified analytical one-degree-of-freedom model is proposed to describe its dynamics both in the linear and nonlinear regimes.

Finally, the theoretical findings are compared with experimental data. A good agreement between the theoretical predictions and the experimental data is found in the static and in the linear dynamic regimes despite the strong hypotheses on the over etch and the simplified one-degree-offreedom model. This validates the proposed auxetic structure and makes it available for future applications in MEMS devices. Comparatively large discrepancies are found when the large displacement regime is entered: this result was expected from all the hypotheses introduced in the modelling. However, the overall softening behaviour of the structure is qualitatively catched by the model.

Future work will be addressed to the theoretical modelling of the nonlinear dynamic behaviour of the proposed structure. A complete numerical code able to combine the electrostatic and mechanical nonlinear phenomena is in fact needed to predict, in a quantitative way, the experimental response of the MEMS structure.

\section{Acknowledgments}

The authors thank STMicroelectronics for the fabrication of the auxetic structure. VZ, RA and AC acknowledge the national project PRIN15 n. 2015LYYXA8.

\section{ORCID iDs}

Valentina Zega (i) https://orcid.org/0000-0003-1607-5827 Marco Ferrari (i) https://orcid.org/0000-0002-3310-7715 Alberto Corigliano (1) https://orcid.org/0000-00021285-2724 


\section{References}

[1] Ruby C D 2012 Poisson's Ratio (Fidel)

[2] Kolken H M A and Zadpoor A A 2017 RSC Adv. 75111

[3] Ren X, Das R, Tran P, Ngo T D and Xie Y M 2018 Smart. Mater. Struct. 27023001

[4] Ungureanu B, Achaoui Y, Enoch S, Brûlé S and Guenneau S 2015 EPJ Appl. Metamater. 28

[5] Alderson A and Alderson K L 2007 Proc. Inst. Mech. Eng. G $221565-75$

[6] Bellis M L D and Bacigalupo A 2017 Smart Mater. Struct. 26 085037

[7] Vadalà F, Bacigalupo A, Lepidi M and Gambarotta L 2018 Compos. Struct. 201 340-51

[8] Bacigalupo A, Lepidi M, Gnecco G and Gambarotta L 2016 Smart Mater. Struct. 25054009

[9] D’Alessandro L, Zega V, Ardito R and Corigliano A 2018 Sci. Rep. 82262

[10] Koenders M A C 2009 Phys. Status Solidi B 246 2083-8

[11] Ma C, Lei H, Hua J, Bai Y, Liang J and Fanga D 2018 Compos. Struct. 203 142-52

[12] Pasternak I S E and Dyskin A V 2012 Int. J. Solids Struct. 49 2239-53

[13] Chen Y and Fu M H 2017 Smart Mater. Struct. 26105029

[14] Fozdar D Y, Soman P, Lee J W, Han L H and Chen S 2011 Adv. Funct. Mater. 21 2712-20

[15] Lantada A D, Muslija A and García-Ruíz J P 2015 Smart Mater. Struct. 24055013

[16] Valente J, Plum E, Youngs I J and Zheludev N I 2016 Adv. Mater. 28 5176-80

[17] Krödel S, Delpero T, Bergamini A, Ermanni P and Kochmann D M 2014 Adv. Eng. Mater. 16 357-63

[18] Stupkiewicz S and Lengiewicz M J L J 2014 Int. J. Solids Struct. 51 3931-43

[19] Goldstein R V, Gorodtsov V A, Lisovenko D S and Volkov M A 2016 Smart Mater. Struct. 25054006

[20] Corigliano A, Ardito R, Comi C, Frangi A, Ghisi A and Mariani S 2018 Mechanics of Microsystems (New York: Wiley)

[21] Alaie $\mathrm{S}$ et al 2010 Thermal transport in micro-scale phononic crystals: Observation of coherent phonon scattering at room temperature and its implications to thermoelectrics Nat. Commun. 67228

[22] Kuo N and Piazza G 2010 Evidence of acoustic wave focusing in a microscale $630 \mathrm{Mhz}$ aluminum nitride phononic crystal waveguide 2010 IEEE Int. Frequency Control Symp. pp 530-3

[23] Olsson R H III, El-Kady I and Tuck M R 2008 Microscale phononic band-gap crystals and devices SAND2008

[24] Han Z, Kohno K, Fujita H, Hirakawa K and Toshiyoshi H 2014 Opt. Express 2221326

[25] Zhu H and E-YLee J 2015 Proc. Eng. 120 516-9

[26] Rottemberg X, Jansen R, Verheyen P, Vanoof R, Verbist A and Tilmans H 2011 Engineering of acoustic metamaterials with application to mems baw resonators TRANSDUCERS 2011

[27] Ardito R, Cremonesi M, D’Alessandro L and Frangi A 2016 Application of optimally-shaped phononic crystals to reduce anchor losses of mems resonators IEEE Int. Ultrasonic Symp. pp 1-3

[28] Bruggi M, Zega V and Corigliano A 2016 Optimization of auxetic structures for mems applications 2016 17th Int Conf. on Thermal, Mech. and Multi-Physics Simulation and Experiments in Microelectronics and Microsystems (EuroSimE) pp 1-5

[29] Bruggi M, Zega V and Corigliano A 2017 Struct. Multidiscip. Optim. 55 1-12

[30] Zhanshe G, Fucheng C, Boyu L, Le C, Chao L and Ke S 2015 Microsyst. Technol. 21 2053-66

[31] Zega V, Langfelder G, Falorni L G and Comi C 2019 J. Microelectromech. Syst. 28 189-98

[32] Srikar V T and Spearing S M 2003 Exp. Mech. 43 238-47

[33] Osterberg P M and Senturia S D 1997 J. Microelectromech. Syst. 6 107-18

[34] Corigliano A, Masi B, Frangi A, Comi C, Villa A and Marchi M 2004 J. Microelectromech. Syst. 13 200-19

[35] Clough R W and Penzien J 1993 Dynamics of Structures (New York: McGraw Hill)

[36] Landau L D and Lifshitz E M 1965 Meccanica (Torino: Boringhieri)

[37] Veijola T, Mattila T, Jakkola O, Kiihamaki J, Lamminmaki T, Oja A, Ruokonen K, Sepa H, Seppala P and Tittonen I 2000 IEEE MTT-S Int. Microwave Symp. Digest vol 1, pp 99-102

[38] Hosen M A, Chowdhury M S H, Ali M Y and Ismail A F 2017 Ital. J. Pure Appl. Math. 37 455-66

[39] Younis M I and Nayfeh A H 2003 Nonlinear Dyn. 31 91-117

[40] Younis M I, Abdel-Rahman E M and Nayfeh A H 2002 Static and dynamic behavior of an electrically excited resonant microbeam Proc. AIAA 43rd Structures, Structural Dynamics, and Materials Conf. pp 1305

[41] Nayfeh A H 1981 Introduction of Perturbation Techniques (New York: Wiley)

[42] Nayfeh A H and Mook D T 1995 Nonlinear Oscillations (New York: Wiley)

[43] Allgower E L and Georg K 1990 Numerical Continuation Methods (Berlin: Springer)

[44] Guerrieri A, Frangi A and Falorni L 2018 J. Microelectromech. Syst. 27 963-72

[45] Frangi A, Guerrieri A, Boni N, Carminati R, Soldo M and Mendicino G 2016 IEEE Trans. Ind. Electron. 65 5962-9

[46] Cerini F, Ferrari M, Ferrari V, Russo A, Urquia M, Ardito R and De Masi B 2017 Sensors Actuators A 266 219-31

[47] Arnau A, García J V, Jimenez Y, Ferrari V, Ferrari M and Sedmik R 2008 Rev. Sci. Instrum. 79075110

[48] Ferrari M and Ferrari V 2009 Meas. Sci. Technol. 20 124005 